\title{
DEVICE ASSOCIATED CANDIDA INFECTION, INTENSIVE CARE UNITS PATIENTS AND ANTIFUNGAL SENSITIVITY WITH SPECIAL CONCERN TO BIOFILM FORMATION
}

\author{
By \\ Ibrahim Shams Fateh Allah Ali, Muhammad Fadl Amin, Mohammad \\ Essayed Abo-Ghabsha, Ahmed Mahmoud Mohamed El-Garhy* \\ Departments of Clinical Pathology and Anaesthesia \& Intensive Care*, Faculty of \\ Medicine, Al-Azhar University \\ Corresponding author: Ibrahim Shams Fateh Allah Ali, \\ E-mail: Ibrahimshams97@gmail.com
}

\begin{abstract}
Background: Candida is a genus of yeasts and is the most common cause of fungal infections worldwide. Candida spp. is the commonest cause of disseminated mycoses with reported mortality to be as high as 40 $50 \%$. They account for about $15 \%$ of total health care associated infections (HCAIs) and more than $72 \%$ of nosocomial mycoses.
\end{abstract}

Objective: This was a descriptive cross-sectional study and aimed to study the spread of device associated candida infection in ICU patients, and ability of candida infection associated with medical devices to form biofilm and multidrug resistance candida infection.

Patients and Methods: The present study was conducted in the Department of Microbiology at El-Hussien Hospital, Cairo, Egypt from September 2019 to January 2020 and included patients who were admitted to ICU for more than $48 \mathrm{~h}$ and were exposed to medical devices.

Results: Number of all patients in ICUs included in the present study was 205 cases. Half of them have no growth $(52.7 \%)$, and the other half has either bacterial (32.7\%), candidal $(12.7 \%)$ or mixed infections $(2 \%)$. Identification of types of candida in device associated infection according to CHRO Magar and API system showed no statistical significant difference. Fluconazole (FLU) was sensitive in 19 patients (63.3\%), Posaconazole (Posa) was sensitive in 22 patients (73.3\%), Voriconazole (Vori) was sensitive in 18 patients $(60 \%)$ and Cuspofungin (Cuspo) was sensitive in 26 patients (86.7\%). According to the sensitivity results in patients with biofilm, Fluconazole (FLU) and Posaconazole (Posa) were sensitive in 2 patients (40\%), while Voriconazole (Vori) and Cuspofungin (Cuspo) were sensitive in 3 patients (60\%).

Conclusion: The increased incidence of systemic mycoses caused by candida infection in hospitalized patients is an important cause of morbidity and mortality worldwide, especially in critically ill patients. Biofilms play an important role in the perpetuation of these infections primarily with respect to their ability to adhere to various medical devices.

Keywords: Candida Infection, Intensive Care Units patients, Antifungal Sensitivity, Biofilm Formation.

\section{INTRODUCTION}

Health care associated infections (HCAIs) or hospital acquired infections
(HAIs) are infections that occur during hospitalization but are neither present nor incubating upon hospital admission. Various factors like increasing incidence 
of hospitalization, rapid advancement in medical technology, and injudicious use of antibiotics along with better adaptation of microbes to the hospital environment contribute to exponential increase in HCAIs (Deorukhkar et al., 2014).

Mycotic pathogens are increasingly reported as causes of HCAIs. Fungi, especially Candida spp., rank 3rd among various leading cause of catheterassociated infections, which confirms the close relation between candida and medical devices (Lockhart, 2014). So, it was a need for reproducible, clinically relevant antifungal susceptibility testing which has been prompted by the increasing number of invasive fungal infections, the expanding use of new and established antifungal agents, and recognition of antifungal resistance as an important clinical problem (Pfaller, 2012).

In considering antifungal susceptibility, it is important to understand that the overwhelming majority of data has been generated through in vitro testing of planktonic Candida cells. As has been well-recognized for bacteria, Candida within biofilms is less susceptible to antimicrobial agents than cells growing under planktonic conditions (Mathé and Van Dijck, 2013).

The balance between $\mathrm{C}$. albicans and non-Candida albicans Candida (NCAC) species determines the profiles associated with virulence. So, the most common species are C. albicans, C. tropicalis, C. glabrata, C.dubliniensis, C.parapsilosis, C.orthopsilosis, C.metapsilosis, C.krusei, C.famata, C.guilliermondii and C.lusitaniae. Other relevant fact associated with virulence is their capacity to form biofilms with other species, which together with the presence of teleomorph forms (sexual phase of fungi in which the same biologic entity could have two different scientific names), difficult the treatment and alter the susceptibility profiles to traditional antifungal agents (Mayer et al., 2013).

$\begin{array}{ccr}\text { Initiation } & \begin{array}{c}\text { involves } \\ \text { organization }\end{array} & \begin{array}{r}\text { cellular } \\ \text { and }\end{array}\end{array}$
differentiation. Sessile yeast cells divide and produce proteins, polysaccharides, extracellular nucleic acids, and lipids that collect and organize at the surface of the growing layer of cells. The cells become immobilized by this dense overlying ECM, which increases cell-to-cell contact and communication (Flemming and Wingender, 2010).

Some Candida cells differentiate from the yeast morphology to filamentous morphologies like hyphae or pseudohyphae. Candida albicans is notable among the medically relevant species for the formation of true hyphae, which is regulated in part by transcription factors like Bcr1 or Tec1 (Bonhomme and d'Enfert, 2013).

Interestingly, the ability of C. albicans to form true hyphae has little impact on the overall metabolic activity of biofilm, as non-C. albicans spp. have been shown to be more metabolically active on the whole (Ferreira et al., 2013).

Hyphal formation by C. albicans during biofilm formation is regulated, at least in part, by quorum sensing. Quorum sensing is the regulation of gene expression by microbes in response to cell density (Yu et al., 2012).

During maturation, the ECM develops into a more extensive structure, and 
changes in Candidagene expression patterns lead to increased drug resistance. For example, efflux pumps may be upregulated, or glucan molecules on the matrix surface may prevent drugs from accessing their targets (Finkel and Mitchell, 2011).

Biofilm structure varies by particular strain and by Candida species. C. glabrata and $\mathrm{C}$. parapsilosis form thinner, more compact biofilms than other species. C. tropicalis forms layers of blastoconidia and a thick outer matrix layer, and $\mathrm{C}$. krusei biofilms manifest as thick structures with a liberal ECM (Costa et al., 2013).

At the point of dispersal, cells are released to the surrounding environment, potentially seeding new sites of infection and leading to disseminated infection (Finkel and Mitchell, 2011).

The present study aimed to study the spread of device associated candida infection in ICU patients, and ability of candida infection associated with medical devices to form biofilm and multidrug resistance candida infection.

\section{PATIENTS AND METHODS}

The present study was conducted in the Department of Microbiology at AlHussien Hospital, Cairo, from September 2019 to January 2020, and included patients who were admitted to ICU for more than $48 \mathrm{~h}$, and were exposed to medical devices.

The following three commonly encountered medical device-associated candidal infections, catheter-associated urinary tract infection (CA-UTI), intravascular catheter-related blood stream infections (CR-BSI), and ventilatorassociated pneumonia (VAP) were targeted, and ethical written informed consents were obtained.

In case of CA-UTI, urine sample was collected aseptically from ICU patients from sampling part of indwelling urinary catheter with sterile syringe and needle. The CR-BSI was suspected when a patient with central venous catheter developed fever or other symptoms of sepsis of unknown origin. In such cases, two blood samples were aseptically collected; the first blood samples was obtained from the catheter itself and the second from the other arm, and were immediately transported to microbiology laboratory. VAP was suspected in a patient on mechanical ventilator when there is a development of new fever, supported by radiological evidence of a new or progressive pulmonary infiltrate and leukocytosis, then bronchoalveolar lavage (BAL), and tracheal aspirate were collected from these patients from endotracheal tubes at morning before routine suction done under aseptic conditions and transported immediately to microbiology unit.

The samples collected from different medical devices in ICU patients were inoculated on blood agar, MacConkey's agar or (cystine-lactose-electrolytedeficient agar (CLED) in case of urine samples) and Sabouraud dextrose agar (SDA) and incubated at $35^{\circ} \mathrm{C}$ for $24-48 \mathrm{~h}$.

Candida clearly appeared on Sabouraud dextrose agar (SDA) like nonshiny whitish colonies and wet film done for all cases of suspected candida for morphological identification assurance then subculture on SDA and on 
autoclaved sterile glycerol $15 \%$ broths done to preserve them for further tests.

Germ tube test was done to identify C.albicans which was still the commonest type of candida worldwide: $1 \mathrm{ml}$ of human serum was put into a small tube. A colony of yeast was gently emulsified in the serum. The tube then Incubated at $37^{\circ} \mathrm{C}$ for 3 hours. A drop from the serum was placed on a slide with a coverslip and examined microscopically under low and high power objectives. Positive Test in C.albicans: A short hyphal (filamentous) extension arising laterally from a yeast cell, with no constriction at the point of origin. Germ tube was half the width and 3 to 4 times the length of the yeast cell. Differentiation between types of candida done by advanced methods:

$1^{\text {st }}$ with CHROMagarTM Candida method which was a type of media that differentiates between types of candida according to the color of colonies growth on this media.

$2^{\text {nd }}$ method via API 20C AUX system (bioMérieux) which was a system for the precise identification of the most frequently encountered candida spp.

Antifungal susceptibility testing was done by disc diffusion method, and four antifungals were chosen which were recommended by CLSI document M44A2 which provided an established methodology for disc diffusion testing of Candida spp zone interpretive criteria for caspofungin, fluconazole, posaconazole and voriconazole and recommended quality control ranges for these four antifungals. Disc diffusion was done on Muller-Hinton Agar+ 2\% glucose and 0.5 microgram $/ \mathrm{ml}$ Methylene blue dye.
Glucose in concentration of $2 \%$ provided suitable fungal growth and the addition of methylene blue dye to concentration of $0.5 \mathrm{microgram} / \mathrm{ml}$ enhanced zone of definition.

Identification of candida forming biofilm was by using Congo Red Agar. Medium this was formed of:

1. Congo red dye 0.8. gm (purchased from ALPHA, Batch No.C0511).

2. Sucrose $36 \mathrm{gm}$.

3. Brain heart infusion agar $52 \mathrm{gm}$ (purchased from OXOID, CM1136).

4. Distal water $1000 \mathrm{ml}$.

Congo red stain was prepared first as concentrated aqueous solution separately from constituents of medium. Congo red was autoclaved at $121 \mathrm{C}$ for 15 minutes. Congo red stain was added to the autoclaved brain heart infusion agar with sucrose which was cooled at 55C.Medium was poured in sterilized plates, covered and left to become cold then stored in the refrigerator $(2-8 \quad$ C). Plates were inoculated and incubated aerobically for 24 to 48 hrs. at $37 \mathrm{C}$. Positive result was indicated by black colonies with adry crystalline consistency.

\section{Statistical analysis:}

Data was fed to the computer and analyzed using IBM SPSS software package version 20.0. Qualitative data were described using number and percent and were compared by Chi-square (x2) test. P-value was considered when the level was $<0.05$. 


\section{RESULTS}

Number of all patients in ICUs included in the present study were 205 cases, around half of them has no growth
$(52.7 \%)$ and the other half has either bacterial $(32.7 \%)$, candidal $(12.7 \%)$ or mixed infections (2\%) (Table 1).

Table (1): Description of all studied patients with variant infections

\begin{tabular}{|c|c|c|c|}
\hline \multirow{2}{*}{ Results } & \multicolumn{2}{c|}{$\begin{array}{c}\text { All admitted patients } \\
\text { (Total= 205 Cases) }\end{array}$} \\
\hline \multirow{4}{*}{ No growth } & 108 & $52.7 \%$ \\
\cline { 2 - 4 } & Bacterial & 67 & $32.7 \%$ \\
\cline { 2 - 4 } & Candidal VAP & 3 & $1.5 \%$ \\
\cline { 2 - 4 } & Candidal BSI & 2 & $1 \%$ \\
\cline { 2 - 4 } & Candidal UTI & 21 & $10.2 \%$ \\
\cline { 2 - 4 } & Mixed infection & 4 & $2 \%$ \\
\hline
\end{tabular}

Identification of types of candida in device associated infection according to CHROMagar and API system shows no statistical significant difference ( $\mathrm{p}$-value > 0.05) (Table 2).

Table (2): Comparison between Chrome agar and API system as regard results

\begin{tabular}{|c|c|c|c|c|c|}
\hline $\begin{array}{ll}\text { Results } & \text { Devices }\end{array}$ & \multicolumn{2}{|c|}{$\begin{array}{c}\text { Chrome agar } \\
(\mathbf{N}=\mathbf{3 0})\end{array}$} & \multicolumn{2}{|c|}{$\begin{array}{l}\text { API Sys } \\
(\mathbf{N}=\mathbf{3 0})\end{array}$} & P-value \\
\hline Albicans & 20 & $66.7 \%$ & 18 & $60 \%$ & \multirow{5}{*}{$0.526 \mathrm{NS}$} \\
\hline Glabrata & 6 & $20 \%$ & 5 & $16.7 \%$ & \\
\hline Albicans + Klebsiella & 3 & $10 \%$ & 3 & $10 \%$ & \\
\hline Albicans + Enterobacter & 1 & $3.3 \%$ & 1 & $3.3 \%$ & \\
\hline Krusei & 0 & $0 \%$ & 3 & $10 \%$ & \\
\hline
\end{tabular}

According to the sensitivity results of all studied patients with candida infections. Fluconazole (FLU) was sensitive in 19 patients $(63.3 \%)$, Posaconazole (Posa) was sensitive in 22 patients $(73.3 \%)$, Voriconazole (Vori) was sensitive in 18 patients $(60 \%)$ and Cuspofungin (Cuspo) was sensitive in 26 patients $(86.7 \%)$. According to biofilm formation in all studied patients. There were 5 patients $(16.7 \%)$ positive and 25 patients $(83.3 \%)$ negative (Table 3). 
Table (3): Description of sensitivity results and biofilm formation in all studied patients

\begin{tabular}{|c|c|c|c|}
\hline \multicolumn{2}{|c}{ Results } & \multicolumn{2}{c|}{$\begin{array}{c}\text { Studied patients } \\
(\mathbf{N}=\mathbf{3 0})\end{array}$} \\
\hline \multirow{2}{*}{ Fluconazole (Flu) } & Sensitive & 19 & $63.3 \%$ \\
\cline { 2 - 4 } & Resistant & 11 & $36.7 \%$ \\
\hline \multirow{2}{*}{ Posaconazole (Posa) } & Sensitive & 22 & $73.3 \%$ \\
\cline { 2 - 4 } & Resistant & 8 & $26.7 \%$ \\
\hline \multirow{2}{*}{ Voriconazole (Vori) } & Sensitive & 18 & $60 \%$ \\
\cline { 2 - 4 } & Resistant & 12 & $40 \%$ \\
\hline \multirow{2}{*}{ Cuspofungin (Cuspo) } & Sensitive & 26 & $86.7 \%$ \\
\cline { 2 - 4 } & Resistant & 4 & $13.3 \%$ \\
\hline \multirow{2}{*}{ Biofilm formation } & Negative & 25 & $83.3 \%$ \\
\cline { 2 - 4 } & Positive & 5 & $16.7 \%$ \\
\hline \multirow{2}{*}{} & & & \\
\hline
\end{tabular}

The 5 patients with biofilm formation were candida Albicans $(100 \%)$ by CHROMagar while by API system there were 4 candida Albicans (80\%) and 1 candida Krusei (20\%) (Table 4).

Table (4): Description of Chrome agar and API system results in patients with biofilm

\begin{tabular}{|c|c|c|c|c|c|}
\hline \multicolumn{2}{|c|}{ Devices } & \multicolumn{2}{c|}{$\begin{array}{c}\text { Chrome agar } \\
(\mathbf{N}=\mathbf{5})\end{array}$} & \multicolumn{2}{c|}{$\begin{array}{c}\text { API Sys } \\
(\mathbf{N}=\mathbf{5})\end{array}$} \\
\hline \multirow{2}{*}{ Results } & Albicans & 5 & $100 \%$ & 4 & $80 \%$ \\
\cline { 2 - 6 } & Krusei & 0 & $0 \%$ & 1 & $20 \%$ \\
\hline
\end{tabular}

According to the sensitivity results in patients with biofilm. Fluconazole (FLU) and Posaconazole (Posa) were sensitive in
2 patients (40\%) while Voriconazole (Vori) and Cuspofungin (Cuspo) were sensitive in 3 patients $(60 \%)$ (Table 5).

Table (5): Description of sensitivity results in patients with biofilm

\begin{tabular}{|c|c|c|c|}
\hline \multicolumn{2}{|c|}{} & \multicolumn{2}{c|}{$\begin{array}{c}\text { Studied patients } \\
(\mathbf{N}=5)\end{array}$} \\
\hline \multirow{2}{*}{ Fluconazole (Flu) } & Sensitive & 2 & $40 \%$ \\
\cline { 2 - 4 } & Resistant & 3 & $60 \%$ \\
\hline \multirow{2}{*}{ Posaconazole (Posa) } & Sensitive & 2 & $40 \%$ \\
\cline { 2 - 4 } & Resistant & 3 & $60 \%$ \\
\hline \multirow{2}{*}{ Voriconazole (Vori) } & Sensitive & 3 & $60 \%$ \\
\cline { 2 - 4 } & Resistant & 2 & $40 \%$ \\
\hline \multirow{2}{*}{ Cuspofungin (Cuspo) } & Sensitive & 3 & $60 \%$ \\
\cline { 2 - 4 } & Resistant & 2 & $40 \%$ \\
\hline
\end{tabular}

\section{DISCUSSION}

Fungal infections are challenging healthcare infections in critically-ill patients due to the difficulty in diagnosis and empirical management. These infections are usually associated with increased rates of morbidity and mortality. Candida species are the third most 
frequent cause of bloodstream infections in ICU and the main clinical presentation is invasive candidiasis (IC). Prolonged length of stay, presence of invasive devices, and high doses of corticosteroids are some conditions associated with Candida infections (Vincent et al., 2009).

In the present study, samples were collected from patients in ICU associated with medical devices. Three types of samples were collected: urine samples from urinary catheter, blood samples from CVP and endotracheal aspirate from endotracheal tubes of patients on mechanical ventilations. All isolates were inoculated on blood agar, MacConkey's agar or (cystine-lactose-electrolytedeficient agar (CLED) in case of urine samples) and Sabouraud dextrose agar (SDA) and incubated at $35^{\circ} \mathrm{C}$ for $24-48 \mathrm{~h}$. and the result was as following:

Of all 205 samples included in this study, $52.7 \%$ were with no growth, while $32.7 \%$ were with bacterial infections. Candida infections represented $14.6 \%$ and mixed infections showed the least one with $2 \%$. Samples with pure candida growth and with mixed infections were identified via CHROMagar and API systems and found that most of cases with device associaited candida infections were with candida albicans with $66.7 \%$ of cases according to CHROMagar and 66\% according to API identification system, then C.glabrata came second ,mixed infections third and C. krusei fourth. These findings were similar to those of other studies from other countries like Cortes et al. (2014) which described C. albicans as the most common fungal species causing candida infections in ICU in two Colombian studies. In contrast,
Lockhart (2014) described that the frequency of $\mathrm{C}$. albicans is decreasing, while the prevalence of $\mathrm{C}$. parapsilosis and $\mathrm{C}$. tropicalis is rising.

Regarding the urine isolates which represented most of cases in the present study (76.7\% of all candida isolates), C. albicans and non-C. albicans Candida species were $(82.6 \%$ vs. $17.4 \%$ according to CHROMagar and $73.9 \%$ vs $26.1 \%$ according to API system), which reflected prevalence of C.albicans in urinary catheter associated candida infections $\mathrm{C}$. glabrata ranked second after $\mathrm{C}$. albicans (17.4\% according to CHROMagar), but showed similarity between C.glabrata and C.krusei according to API identification system (13\% of each). Gabriel et al. (2017) described C. albicans and non-C. albicans Candida species were similar (54\% vs. $45 \%)$, and C. glabrata ranked third, after C. albicans and C. tropicalis. According to Deorukhkar et al. (2014), C. tropicalis is the most common cause of nosocomial Candida BSI in India.

Susceptibility testing of samples with candida growth for the four recommended antifungals by CLSI done via disc diffusion method on modified MullerHinton and showed that C. albicans (according to API identification system) represented most Susceptibilities to cuspofungin $(81.8 \%)$ and posaconazole $(77.2 \%)$ and less susceptibility to voriconazole $(63 \%)$ and fluconazole (68.2\%). Nucci et al. (2013) showed, in contrast, high Susceptibilities of C. albicans, to fluconazole $(95 \%)$ and voriconazole $(96 \%)$.

C.glabrata (according to API system) came after C.albicans with $(16.7 \%)$ of total candida isolates and showed very 
high susceptibility to cuspofungin (100\%), least susceptibility to voriconazole (20\%) and moderate susceptibility to posaconazole $(60 \%)$ and fluconazole (40\%). In contrast, the susceptibility rate of C. glabrata to fluconazole was high (92.1\%) according to Nucci et al. (2013).

C. krusei came third in this study with $10 \%$ of total candida isolates (according to API system), but no C. krusei isolates found via (CHROMagar system of identification).API system was more accurate than CHROMagar, $(66.7 \%)$ of $\mathrm{C}$. krusei cases were sensitive while just one third of C. krusei cases were resistant to fluconazole.But if results depended just on CHROMagar so, we cannot talk about C.krusei as no cases of it were isolated. Biofilm formation testing done on Congo red agar media showed that the majority of all candida isolates did not form biofilm $83.3 \%$, while $16.7 \%$ were positive for biofilm formation.

Most of fomed candidal biofilms were by C.albicans $(100 \%$ according to CHROMagar) but according to API system $80 \%$ by C.albicans and the other $20 \%$ occurred by C.krusei and this was similar to results of Harriott and Noverr (2011).

In contrast, Marak and Dhanashree (2018) found that more than half of candida biofilms caused by non C.albicans $(57.1 \%),(22.4 \%)$ of them formed by C.krusei while C.albicans formed only $(42.9 \%)$ of total biofilms of candida.

Concerning to susceptibility of biofilm formating candidal infections to different antifungals the present study revealed that (40\%) of cases were resistant to all four recommended antifungals, while (20\%) were sensitive to all previous antifungals and the last (40\%) were variable. And if we talk about every antifungal of the four recommended by (CLSI) individually, so cuspofungin and voriconazole showed the highest susceptibility $(60 \%)$, while fluconazole and posaconazole showed the lowest susceptibility.

These previous results revealed no clear correlation between biofilm formation and susceptibility to antifungals in the present study which was concluded also by Marak and Dhanashree (2018). Other more studies found a strong correlation between biofilm formation and the resistance to different antifungals like Maiolo et al. (2016).

\section{CONCLUSION}

The increased incidence of systemic mycoses caused by Candida infection in hospitalized patients is an important cause of morbidity and mortality worldwide, especially in critically ill patients. Biofilms play an important role in the perpetuation of these infections primarily with respect to their ability to adhere to various medical devices.

\section{REFERENCES}

1. Bonhomme $J$ and d'Enfert $C$ (2013): Candida albicans biofilms: building a heterogeneous, drug-tolerant environment. Curr Opin Microbiol., 16(4):398-403.

2. Cortes JA, Reyes P, Gomez CH, Cuervo SI, Rivas $P$ and Casas CA (2014): Clinical and epidemiological characteristics and risk factors for mortality in patients with candidemia in hospitals from Bogota, Colombia. Braz J Infect Dis., 11: 73-85.

3. Costa AC, Pereira CA, Freire $F$ and Junqueira JC (2013): Methods for obtaining reliable and reproducible results in studies of Candida biofilms formed in vitro. Mycoses., 56(6):614-22. 
4. Deorukhkar SC, Saini $S$ and Mathew S (2014): Non-albicans Candida infection: an emerging threat. Interdisciplinary Perspectives on Infectious Diseases, Article ID 615958: 7.

5. Ferreira AV, Prado CG, Carvalho RR, Dias KS and Dias AL (2013): Candida albicans and non-C. albicans Candida species: comparison of biofilm production and metabolic activity in biofilms, and putative virulence properties of isolates from hospital environments and infections. Mycopathologia, 175(3-4):265-72.

6. Finkel JS and Mitchell AP (2011): Genetic control of Candida albicans biofilm development. Nat Rev Microbiol, 9(2):109-18.

7. Flemming $\mathbf{H C}$ and Wingender $\mathbf{J}$ (2010): The biofilm matrix. Nat Rev Microbiol., 8(9):62333.

8. Gabriel M, Muñoz JS, Oñate J, Pallares CJ, Hernández C and Villegas MV (2017): Epidemiology of Candida isolates from Intensive Care Units in Colombia from 2010 to 2013. Revista Iberoamericana de Micología, 34(1): 17-22.

9. Harriott MM and Noverr MC (2010): Ability of Candida albicans mutants to induce Staphylococcus aureus vancomycin resistance during polymicrobial biofilm formation. Antimicrob Agents Chemother., 54(9): 374655.

10. Lockhart SR (2014): Current epidemiology of Candida infection. Clinical Microbiology Newsletter, 36(17): 131-136.

11. Maiolo EM, Oliva A, Tafin UF, Perrotet N, Borens $O$ and Trampuz A (2016): Antifungal activity against planktonic and biofilm Candida albicans in an experimental model of foreignbody infection. J Infect, 72(3):386-92.
12. Marak MB and Dhanashree B (2018): Antifungal Susceptibility and Biofilm Production of Candida spp. Isolated from Clinical Samples. Int J Microbiol., 14(11): 749752.

13. Mathé $L$ and VanDijck $P$ (2013): Recent insights into Candida albicans biofilm resistance mechanisms. Curr Genet., 59(4):251-64.

14. Mayer FL, Wilson D and Hube B (2013): Candida albicans pathogenicity mechanisms. Virulence, 4:119-28.

15. Nucci M, Queiroz-Telles F, AlvaradoMatute T, Tiraboschi IN, Cortes $J$ and Zurita J (2013): Epidemiology of candidemia in Latin America: a laboratory-based survey. PLoS One, 8(3):e59373.

16. Pfaller MA (2012): Antifungal drug resistance: mechanisms, epidemiology, and consequences for treatment. Am J Med., 125:37.

17. Vincent JL, Marshall J, Silva E, Martin CD, Rello DJ, Moreno R and Anzueto A (2009): International study of the prevalence and outcomes of infection in intensive care units: JAMA., 302 (21): 2323-2329.

18. Yu LH, Wei X, Ma M, Chen XJ and Xu SB (2012): Possible inhibitory molecular mechanism of farnesol on the development of fluconazole resistance in Candida albicans biofilm. Antimicrob Agents Chemother., 56(2): 770-5. 


\section{IBRAHIM SHAMS FATEH ALLAH ALI et al.,}

\section{العدوي الفطربه المصاحبة للأجهزة الطبيه بمرضي العنابه المركزه و حساسيتها لمضادات الفطريات مع الإشارة إلى تكوبن البيوفيلم}

إبراهيم شمس فتح الله علي, محمد فضل أمين, محمد السيد أبوغبشه, أحمد محمود محمد محمد الجارحي*

قسمي الباثولوجيا الإكلينيكية, والتخدير والرعاية المركزة*، كلية الطب، جامعة الأزهر

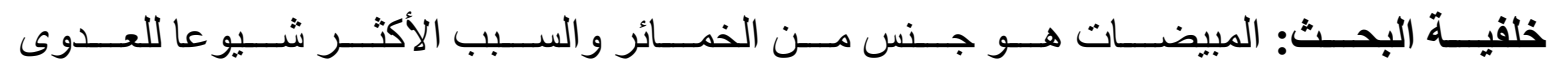

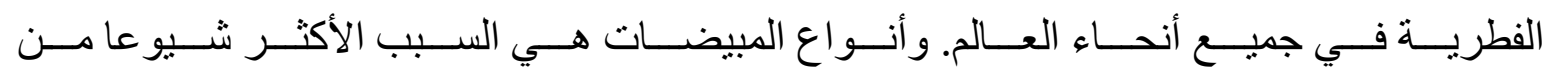

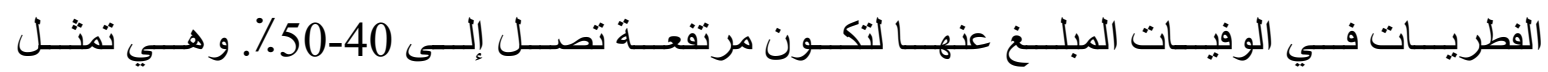

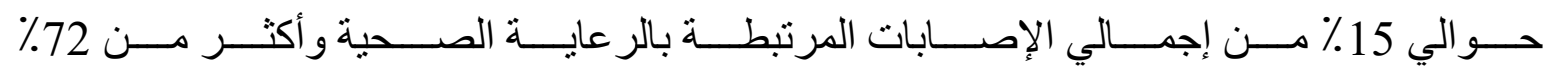
من عدوي الفطريات المرتبطه بالمستشفيات.

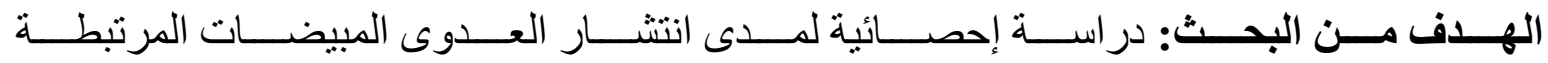

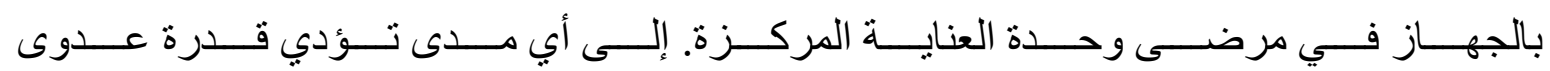

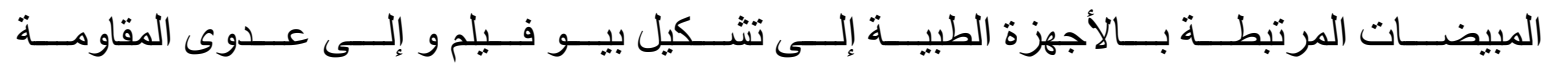
متعددة الأدوية المبيضات.

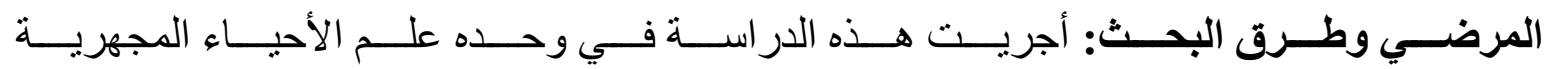

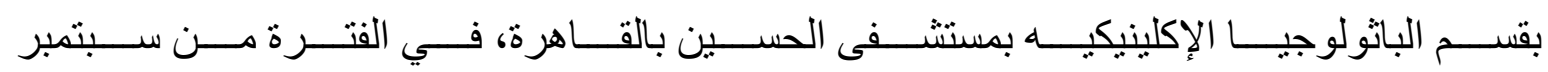

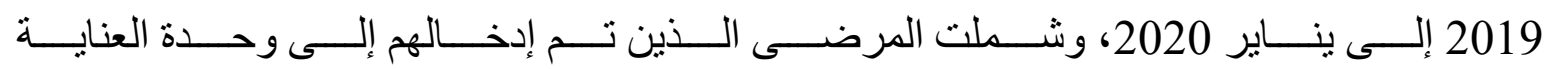
المركزة لأكثر من 48 ساعة وتعرضو اللأجززة الطبية.

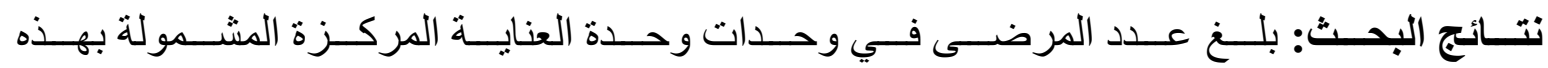

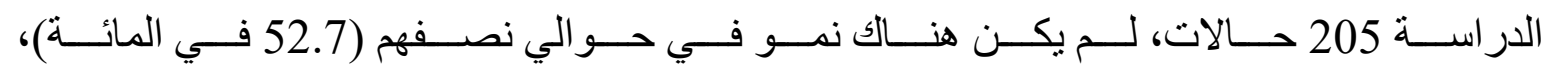

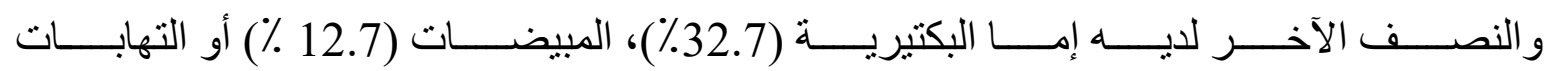

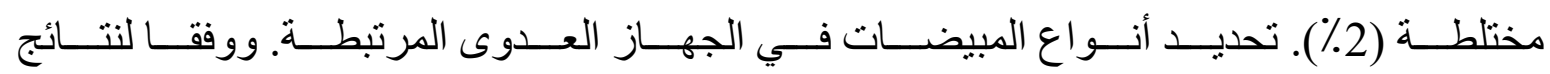

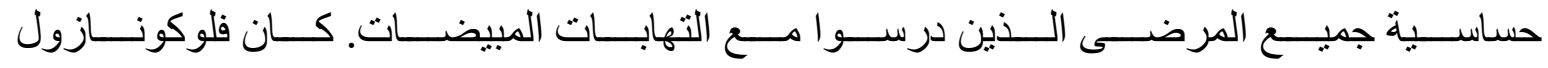

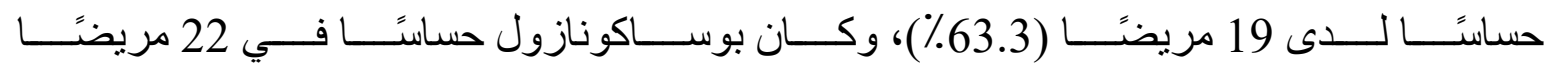

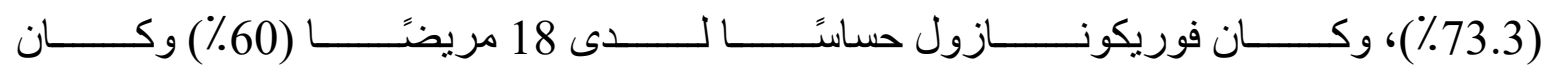




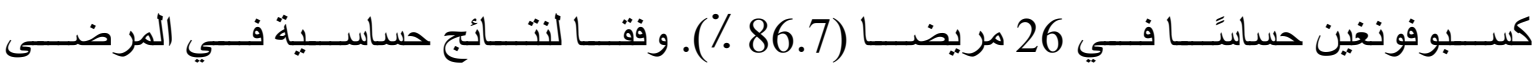
الـــين يعـانون مــن بيــو فــيلم كـان فلوكونــازول وبوســـاكونازول حساسـين فـي مريضــين

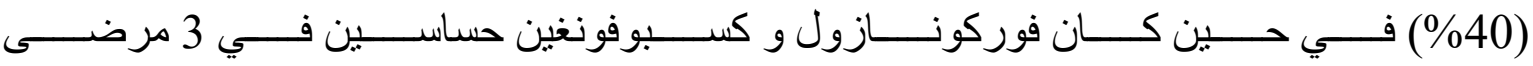

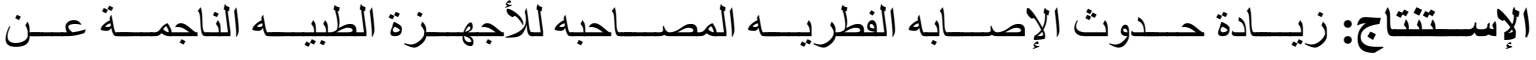

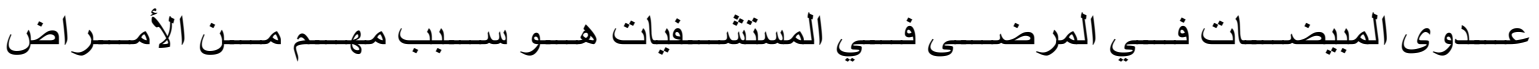

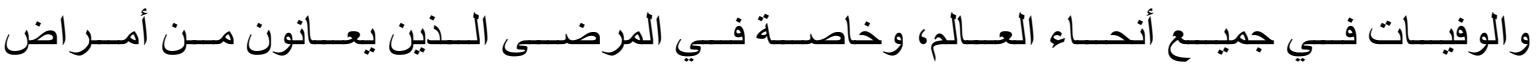

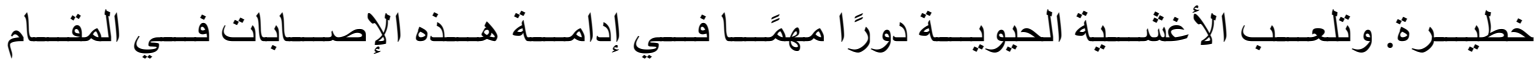
الأول فيما يتعلق بقدرتها على الالتز ام بمختلف الأجهزة الطبية. 